\title{
Evaluación preliminar de la gestión ambiental en hoteles del destino turístico de Varadero, Cuba
}

\section{Resumen}

Laureano José López Moreda* Centro de Servicios Ambientales de Matanzas, Cuba

Elva Esther Vargas Martínez Universidad Autónoma del Estado de México

La aplicación de los sistemas de gestión y certificación ambiental ha cobrado auge internacional en las últimas décadas. La actividad turística, y en particular la hotelera, ha sido abanderada de estas iniciativas. En este artículo se demuestra que la gestión ambiental de las empresas hoteleras estudiadas en Varadero, Cuba, poseen aspectos positivos, asociados a la disposición de cumplir y a las acciones de gestión, así como oportunidades de mejoras relacionadas al monitoreo ambiental y la comunicación. Los resultados individuales permiten clasificar las entidades en las categorías de hotel Líder, Proactivo, Reactivo y Pasivo-indiferente, predominando los dos primeros grupos.

\section{Palabras clave}

Certificación ambiental, gestión ambiental, hoteles, turismo.

Recibido: $18 / 10 / 2012 \cdot$ Aceptado: $22 / 11 / 2012$ 


\title{
Preliminary Evaluation of Environmental Management in Hotels in the tourist destination of Varadero, Cuba
}

Laureano José López Moreda Centro de Servicios Ambientales de Matanzas, Cuba

Elva Esther Vargas Martínez Universidad Autónoma del Estado de México

\begin{abstract}
The use of management systems and environmental certification has acquired an international boom in recent decades. Tourism -and the hotel industry in particular- has had the leading role on these initiatives. This paper shows that the environmental management of the hotel industry studied in Varadero, Cuba, has positive aspects in terms of the actions taken to meet environmental management and to the opportunities related to the enhancement of environmental monitoring and communication. Individual results allow classifying the hotels as 'leader', 'proactive', 'reactive' and 'passiveindifferent', the two latter being dominant.
\end{abstract}

\section{KeY WORDS}

Environmental certification, environmental management, hotels, tourism. 


\section{Introducción}

La gestión ambiental empresarial (GAE) se presenta en la actualidad como una alternativa para enfrentar el deterioro global del ambiente desde la perspectiva local. De estos presupuestos no puede exceptuarse el sector de la hotelería, pues, según Meyer, Aguilera y Boto (2007: 3), “el ambiente no es una cuestión marginal dentro del negocio turístico que pueda resolverse a base de buenas palabras, es el escenario en el que se va a desarrollar buena parte de la competitividad futura del sector".

En Cuba, donde el turismo ha emergido como una de las principales fuentes de ingresos para la economía nacional, los estudios acerca de los impactos ambientales del sector adquieren especial significación, por lo que se han convertido en una prioridad estratégica.

En La Habana y Varadero se concentra 93 \% de la capacidad hotelera del país (Pérez, 2010). En este contexto es necesario desarrollar estudios para crear una plataforma que permita comparar los resultados alcanzados por diferentes organizaciones del sector en su gestión ambiental (GA). De esta forma se crearía una base informativa para respaldar la planificación ambiental, tanto del sector hotelero nacional como de los territorios que acogen destinos turísticos. Por lo anterior, este trabajo se traza como objetivo evaluar los resultados de la GA en los hoteles del destino turístico de Varadero.

\section{Gestión ambiental y gestión ambiental empresarial. Aplicación a las empresas hoteleras}

En Cuba, la Ley 81 del Medio Ambiente considera la gestión ambiental (GA) como "el conjunto de actividades, mecanismos, acciones e instrumentos, dirigidos a garantizar la administración y el uso racional de los recursos naturales mediante la conservación, mejoramiento, rehabilitación, monitoreo del medio ambiente y el control de la actividad del hombre en esta esfera”.

La literatura menciona que la GA, desde el ámbito público, se implementa mediante instrumentos como la política, la legislación y el sistema administrativo (Lascurain, 2006; Sil, 1997). La legislación cubana reconoce además la evaluación del impacto ambiental, el ordenamiento, la inspección ambiental estatal y la educación ambiental. 
La planificación ambiental juega un rol principal dentro de la GA, y los sistemas de gestión ambiental (SGA) son su herramienta fundamental en las empresas (Mateo, 2002). Ellos son definidos, dentro del sistema de gestión gerencial de una organización, como la parte que define la estructura administrativa, las responsabilidades, las prácticas, los procedimientos y los procesos para llevar a cabo la política ambiental (Marques de Almeida y Da Costa, 2002). En la práctica consiste en una revisión de la situación ambiental para identificar, evaluar y controlar los riesgos en cuestiones relacionadas con la naturaleza y ofrecer posibles alternativas a estos (Pereira, 2007; Senior et al., 2007).

Los SGA se complementan con el tratamiento y reciclaje de residuos, las auditorías ambientales, los análisis de ciclo de vida y riesgo, la contabilidad ambiental, los estudios de impacto, los ecobalances, las tecnologías limpias o las evaluaciones del desempeño y los indicadores ambientales (Negrao, 2000; OEA, 2003).

Mercado (2008) señala la incidencia de tres componentes en la GA de las empresas, ellos son: las acciones de gestión ambiental, donde se integra la organización y la trayectoria del desempeño; el influjo de las políticas y la responsabilidad social empresarial, y la exigencia gubernamental y el cumplimiento de la legislación. Por su parte, González y González (2005) resaltan las prácticas logísticas de organización y planificación, así como las de comunicación. A lo anterior incorporan la presión que ejercen los grupos de interés (clientes, organismos reguladores, empleados, prensa, organismos no gubernamentales) sobre las empresas (González y González, 2008).

En el sector turístico, la GAE fue acogida a principios de los setenta (Guevara, 2005). Actualmente, gracias a los preceptos del desarrollo sustentable, la hotelería reconoce su posición frente al deterioro ambiental y busca la manera de mitigar sus impactos negativos y prevenirlos (Llull, 2003).

En los hoteles, la GA se define como aquella que fomenta un uso eficiente de todos los recursos (energía, agua, materias primas, insumos y equipos en general) y se refleja en una operación más limpia y servicios cada vez más amigables con el ambiente (De Burgos y Céspedes, 2004; Guzmán, 2005).

Dentro de las iniciativas voluntarias aplicadas a la GA de los hoteles resaltan los códigos de buenas prácticas ambientales, los programas de auditoría, las ecoetiquetas, los sistemas de certificaciones y otros códigos de autorregulación 
(Yunis, 2003). Estas empresas pueden adoptar los SGA según la Iso 14001, el Sistema Comunitario de Gestión y Auditoría Medioambientales (EMAs, por sus siglas en inglés) o la Carta para el Desarrollo Sostenible de la Cámara Internacional de Comercio (Yunis, 2003; Ayuso, 2003).

De Burgos y Céspedes (2004) reconocen el consenso en la literatura sobre la idoneidad de la auditoría ambiental para medir la efectividad de la GAE. Sin embargo, señalan que, del universo de hoteles que han manifestado su interés en los aspectos ambientales, menos de la mitad la realizan.

Un referente teórico metodológico para evaluar la GAE lo constituye la norma Iso 14031, dirigida a la evaluación del desempeño ambiental, que considera tres dimensiones de análisis: el desempeño operacional, los esfuerzos de gestión y la situación ambiental del entorno de las organizaciones. Esta norma no constituye una guía práctica y no define indicadores ni métodos de evaluación.

Contribución significativa a esta temática es el resultado de Puente (2010), quien propuso un cuestionario para evaluar la aplicación de los instrumentos de la GAE en Cuba. Al evaluar la GA en los hoteles, López (2011) propone un grupo de indicadores agrupados en las dimensiones de educación, comunicación e información ambiental, infraestructura, control operacional, control gerencial, proveedores y consumo responsable.

No obstante, representa la contribución metodológica principal para este texto el trabajo de Vargas, Zizumbo y Viesca (2011), donde se evalúa la GA del sector hotelero en el destino mexicano de Cancún mediante un sistema de indicadores articulados en cuatro dimensiones: acciones de GA, comunicación y consumo responsable, disposición a cumplir y monitoreo.

\section{Caracterización general del destino turístico de Varadero}

El destino turístico de Varadero se localiza en la península de Hicacos, en la costa norte de la provincia de Matanzas, en la región occidental de Cuba, a 140 kilómetros de la ciudad de La Habana. Es el principal balneario del país. Cuenta con más de 23 kilómetros de playas, tiene un aeropuerto internacional y un variado equipamiento turístico. La planta hotelera actual incluye más de 16 700 habitaciones en 53 instalaciones hoteleras, $70 \%$ de las cuales posee categoría de cuatro y cinco estrellas; $89 \%$ de los hoteles es propiedad de empresas nacionales, como Inmobiliaria del Ministerio de Turismo (70 \%) e Inmobiliaria 
Almest (19\%); mientras que $11 \%$ es de capital mixto. La operación recae en cuatro cadenas hoteleras nacionales: Gaviota (10\% de las instalaciones), Cubanacán (13 \%), Gran Caribe (18 \%) e Isla Azul (11 \%). Estas han suscrito contratos de administración con cadenas extrajeras en $64 \%$ de las instalaciones (Mintur, 2012).

Desde el punto de vista ambiental, el destino sobresale por la calidad del aire, del agua de consumo humano y del uso recreativo (playa), así como por la depuración de las aguas residuales. La playa cuenta con una elevada calidad estética, aunque sus condiciones morfodinámicas ${ }^{1}$ se han visto afectadas porque en el pasado, incorrectamente, se ubicaron construcciones sobre las dunas costeras, por la extracción deliberada de arena y por la influencia de fenómenos meteorológicos extremos. Ante esta problemática se realizan acciones periódicas de mantenimiento mediante la tecnología de alimentación artificial, y desde 2002 funciona en el destino el Programa de Manejo Integrado Costero.

Las instalaciones hoteleras poseen como promedio más de diez años de explotación, lo que atenta contra su estado constructivo y de los sistemas técnicoingenieriles. Ello repercute en altos niveles de consumo de agua y energía por turistas/días. Además generan altos volúmenes de residuos sólidos, lo que en un espacio de tanto valor estético-escénico ha motivado iniciativas que potencien el reciclaje.

\section{La gestión ambiental en las empresas hoteleras en el destino turístico de Varadero, Cuba}

El artículo 14 de la Ley 81 del Medio Ambiente preceptúa el carácter obligatorio de medidas y programas para la protección del entorno en el universo empresarial nacional, a pesar de ello, la GAE no es reconocida como un instrumento de esta ley, tampoco de la Estrategia Ambiental Nacional (EAN) como principal expresión de la política ambiental del Estado. Sin embargo, según el Decreto 281, la GAE se ha incorporado como un subsistema del proceso de perfeccionamiento empresarial desarrollado en el país, donde el sector hotelero está ampliamente representado (López y Ramírez, 2011).

${ }^{1}$ Cambios que sufren las playas por la acción de las principales dinámicas que actúan sobre ellas: oleaje, corrientes y vientos (Vidal et al., 1995: 55). 
Cuba cuenta también con una vasta legislación ambiental aplicable a las empresas hoteleras que, unida al proceso de planificación estratégica ambiental desarrollado a partir de la Resolución 40/2007 del Ministerio de Turismo (Mintur), donde se establece la estrategia ambiental del sistema de turismo en el país, conforma un modelo de gestión obligatorio que involucra a los hoteles en la planificación de recursos y acciones para mitigar sus impactos negativos y mejorar su desempeño ambiental. Además, el país desarrolla ampliamente dos iniciativas voluntarias: el Reconocimiento Ambiental Nacional (RAN) y el aval ambiental, otorgado por las autoridades ambientales y asociado al proceso de categorización hotelera. Asimismo, los hoteles pueden optar por la certificación de su sGa por la norma cubana iso 14001 (López y Ramírez, 2011).

\section{Metodología}

El trabajo se apoya en métodos como la revisión documental y el estudio empírico, además de instrumentos como la encuesta, la entrevista a profundidad y las técnicas de estadística descriptiva.

La investigación se realizó entre 2010 y 2011. Contó con un diseño experimental-transversal, pues mediante este se explican las condiciones en que permanecen las empresas, al recolectar los datos durante un periodo de tiempo sin manipular las variables que intervienen en la investigación.

Las cuatro dimensiones que caracterizan la GA y los 24 indicadores utilizados fueron los propuestos por Vargas, Zizumbo y Viesca (2011). El cuestionario se validó mediante la consulta a siete expertos: tres profesores universitarios con doctorado y experiencia en GA, dos consultores ambientales con más de diez años en la actividad y dos especialistas del sector empresarial hotelero. La fiabilidad del cuestionario se determinó mediante el coeficiente Alpha de Cronbach (0.823).

La evaluación se realiza en una escala nominal cualitativa donde el criterio (+) significa que el indicador se aplica y el criterio (-) que no se aplica. Después se determinaron los valores porcentuales $\left(\mathrm{PD}_{(\mathrm{i})}\right)$ de las respuestas positivas $(+)$ para cada (i) dimensión $\left(\mathrm{D}_{(\mathrm{i})}\right)$ en el conjunto de los indicadores de los hoteles muestreados. 


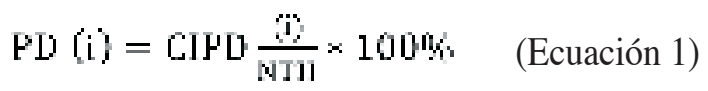

Donde:

CIPD: Cantidad de indicadores dentro de una (i) dimensión con una evaluación positiva en la muestra de hoteles.

NTH: Número total de hoteles en la muestra.

La valoración cualitativa de los resultados de la tipología de GA empleó la propuesta de Azqueta et al. (2007), que ubica los hoteles en las categorías "líder", "proactivo", "reactivo", "pasivo-indiferente” y "negativo", para lo cual se construye una escala de intervalos mediante el método de amplitud y rango. El número de intervalos considerados es la cantidad de categorías en las que se clasificará la gestión (cinco). El rango (R) es determinado según (ecuación 2).

$$
\mathrm{R}=\text { (máx PI) - (mín PI) = } 100 \text { (Ecuación 2) }
$$

Donde:

R: rango.

PI: porcentaje de la aplicación de todos los indicadores en su conjunto (máximo 100 y mínimo 0).

La amplitud (C) de cada uno de los intervalos se determina según (ecuación 3).

$$
\mathrm{C}=\mathrm{R} / \mathrm{K}=100 / 5=20 \text { (Ecuación 3) }
$$

Donde:

K: Es el número de categorías a considerar.

Según el valor de $\mathrm{C}$, se establecen las escalas cualitativas que aparecen en el cuadro 1.

CuAdRo 1. Escala de evaluación cualitativa para los valores alcanzados

en el cuestionario

\begin{tabular}{ccc} 
Intervalo & Criterios para clasificar los hoteles & (Categorías $(\mathrm{k})$ ) \\
$0 \leq \mathrm{PI}<20$ & Negativo & 1 \\
$20 \leq \mathrm{PI}<40$ & Pasivo-indiferente & 2 \\
$40 \leq \mathrm{PI}<60$ & Reactivo & 3 \\
$60 \leq \mathrm{PI}<80$ & Proactivo & 4 \\
$80 \leq \mathrm{PI}<100$ & Líder & 5 \\
\hline
\end{tabular}


Posteriormente se determina el por ciento PJC(j, k) de aplicación de cada (j) indicador para los hoteles incluidos dentro cada categoría (k) (ecuación 4).

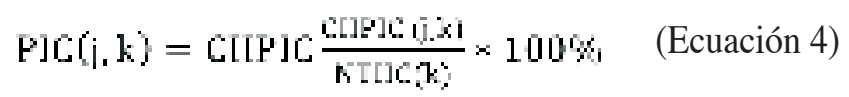

Donde:

$\mathrm{CHPIC}_{(\mathrm{j}, \mathrm{k})}$ : Cantidad de hoteles con evaluación positiva de cada (j) indicador evaluado en los hoteles incluidos dentro de cada (k) categoría.

$\mathrm{NTHC}_{(\mathrm{k})}$ : Número total de hoteles incluidos en cada (k) categoría.

Las respuestas de los cuestionarios aplicados fueron complementadas con entrevistas a profundidad a los directivos, lo que permitió recoger información cualitativa adicional.

La muestra se seleccionó de forma no probabilística, ya que se solicitó la participación de todos los hoteles que integraron el universo de estudio (53). Como resultado de este proceso se obtuvo la colaboración de 14 hoteles, aproximadamente $26 \%$ de la planta hotelera. Las características de la muestra se recogen en el cuadro 2 .

CuAdro 2. Características de la muestra

Destino

Turismo

Modalidad hotelera

Categoría de los hoteles

Tamaño de la muestra respecto a la población de estudio

Existencia de un marco regulatorio de referencia

Existencia de mecanismos nacionales de certificación ambiental

Participación en el estudio Antigüedad
Varadero

Sol y playa

Todo incluido

3,4 y 5 estrellas

$\sim 26 \%$

Sí

Sí

Voluntaria

5-15 años 


\section{Resultados}

De las instalaciones evaluadas, $86 \%$ son de propiedad nacional y solo dos de capital mixto. La explotación es realizada en $57 \%$ por la cadena Cubanacán y 43 \% por Gran Caribe, en 86 \% de los hoteles la administración incluye contratos con grupos hoteleros extranjeros, y solo dos son administrados íntegramente por entidades nacionales. En general superan los diez años de explotación, lo que en ocasiones se evidencia en el deterioro de la infraestructura y el equipamiento hotelero. Un análisis global de los resultados de la gestión según las dimensiones consideradas se puede apreciar en la figura 1 .

D4. Disposición a cumplir

D3. Monitoreo ambiental

D2. Comunicación y consumo responsable

D1. Acciones de GA

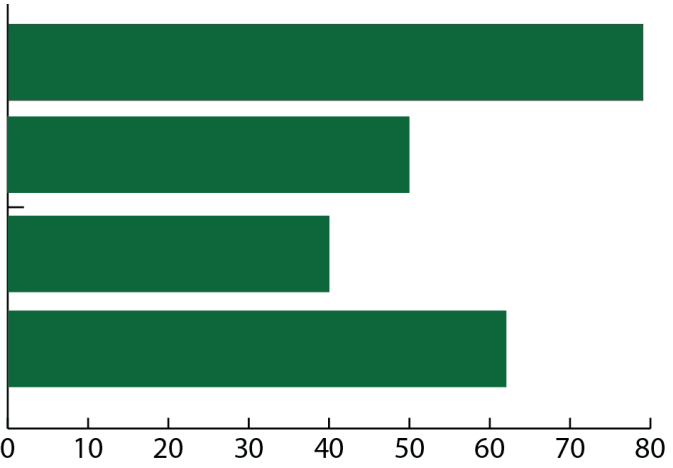

FIgURA 1. Resultados globales de evaluación de los hoteles

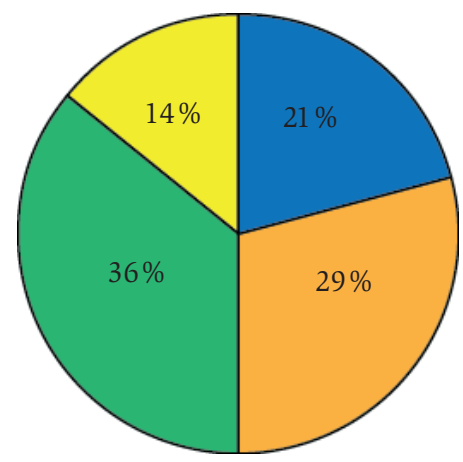


De acuerdo con la tipología de conductas ambientales propuesta por Azqueta (2007), los hoteles estudiados se ubican en las categorías "líder", "proactivo", "reactivo" y "pasivo-indiferente" (figura 2).

En la figura 3 se pueden observar los resultados obtenidos en los indicadores evaluados, agrupados dentro de cada una de las categorías en que fueron clasificados los hoteles de acuerdo con su gestión.

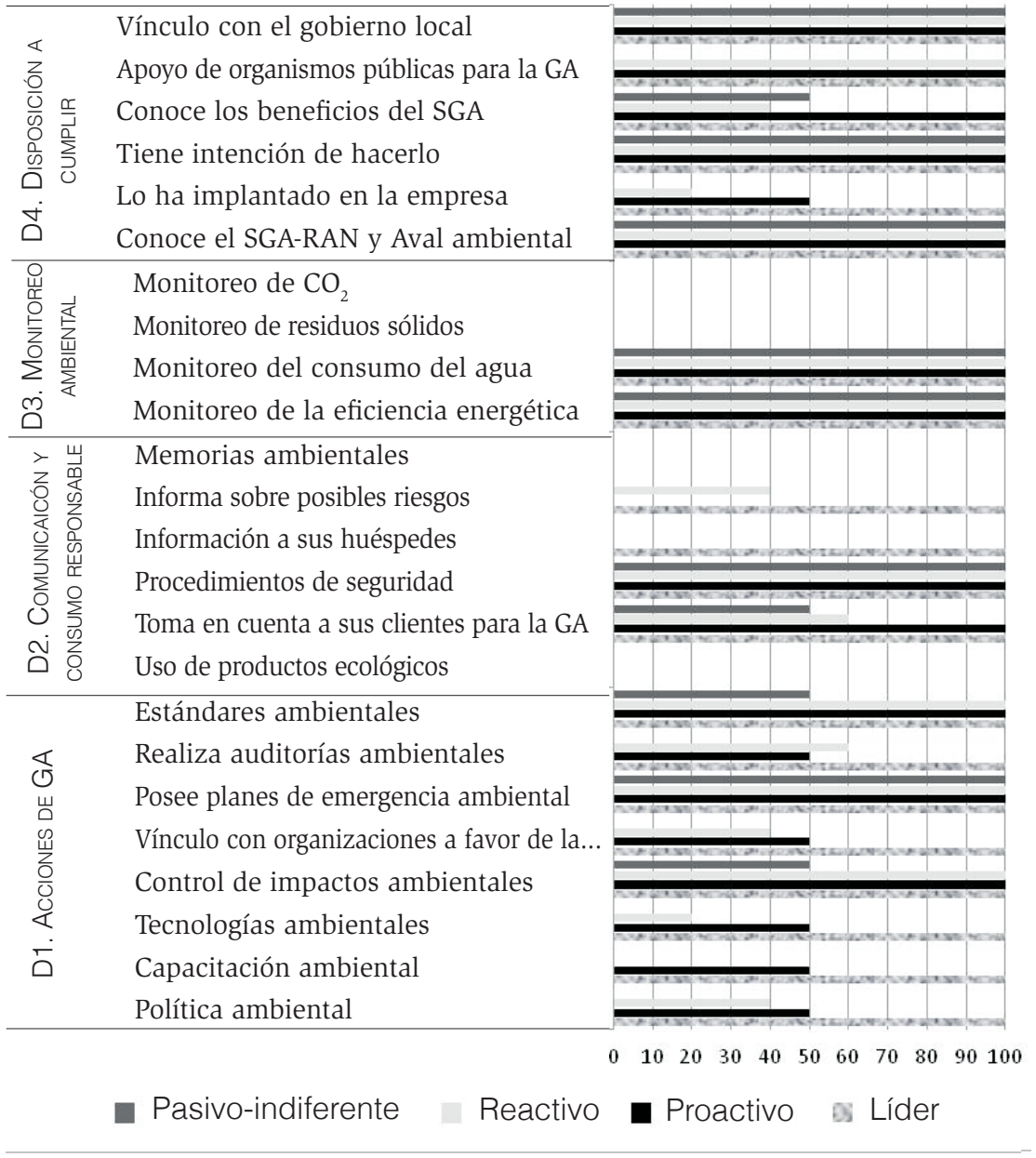




\section{Discusión de resultados}

En general, los hoteles reconocen sus impactos ambientales negativos, identifican sus problemáticas y trazan programas de gestión, pero no siempre cuentan con un respaldo de recursos financieros, materiales y humanos. Los aspectos ambientales más reconocidos son los consumos de energía, agua, emisión de residuos y actuación en la zona costera. El control de los resultados recae principalmente en la inspección ambiental estatal y en las evaluaciones de impacto ambiental, no obstante, el total de la muestra se ha sometido a una auditoría de diagnóstico.

Todos los hoteles han puesto en práctica procedimientos para lograr un mejor desempeño ambiental y controlar sus impactos. Los aspectos más abordados son el manejo de residuos sólidos y peligrosos y la reducción de los consumos de agua, energía y combustibles de todo tipo. Se implementan además acciones dirigidas a la seguridad y capacitación en la aplicación de planes de contingencia ante fenómenos naturales, incendio y otros accidentes.

Entre las principales medidas adoptadas para mitigar los problemas ambientales están el tratamiento de las aguas residuales acorde con las normativas nacionales, el uso de interruptores eléctricos de presencia o accionados por tarjetas o timer, el riego por aspersión o por goteo y concentrado en las primeras horas del día, la sustitución del mobiliario sanitario por otro de menor consumo de agua, la introducción de grifos ahorradores con reductores de flujo y el uso de lámparas eléctricas de bajo consumo. Igualmente, se trabaja en la clasificación y reciclaje de residuos sólidos, así como la segregación y tratamiento adecuado de residuos peligrosos. Sin embargo, estas prácticas se concentran en la reducción de los costos de operación o en una mayor calidad en el servicio, elementos donde se enfoca el interés de los empresarios en los temas ambientales. Además no están extendidas en el total de los hoteles evaluados.

En general, las mayores fortalezas de los hoteles estudiados (figura 1) son las acciones de GA y la disposición a cumplir, y, más limitadas, las acciones de monitoreo ambiental, de comunicación y consumo responsable.

Dentro de la acciones de gestión, la elaboración de la política ambiental del hotel, la capacitación ambiental y la introducción de las mejores tecnologías ambientales no sobrepasan 40 \% de aplicación. Contrastan favorablemente con ello las acciones de control de impactos, el reconocimiento e implantación de 
estándares ambientales en las operaciones y la realización de auditorías.

La disposición a cumplir solo alcanza un valor inferior a $50 \%$ en cuanto a la implementación de un SGA en los hoteles; sin embargo, el conocimiento acerca de los programas de certificación existentes en el país y sus beneficios, así como la disposición a implantarlos y los vínculos con el gobierno local obtuvieron elevadas calificaciones, superiores a $60 \%$.

En cuanto a la comunicación y consumo responsable hay que señalar que es donde hubo resultados más negativos, pues incide mayormente la ausencia de un estímulo por el consumo de productos ecológicos, la no publicación de memorias ambientales y la carencia de información sistemática a los huéspedes acerca de cómo reducir sus impactos negativos y los riesgos ambientales, todos con valores inferiores a $50 \%$ de aplicación. Solamente se alcanzan resultados relevantes en esta dimensión en lo referido a la existencia de mecanismos de retroalimentación acerca de los criterios de los huéspedes y los procedimientos para enfrentar situaciones de emergencia.

Respecto al monitoreo ambiental, los hoteles estudiados monitorean sistemáticamente sus consumos de agua y energía, pero no la generación de residuos sólidos ni las emisiones de $\mathrm{CO}_{2}$.

En lo referente a la clasificación de los hoteles según su GA, el comportamiento es positivo (figura 2), pues $50 \%$ de ellos es líder o proactivo. No obstante, predominan los reactivos, lo cual está acorde con el criterio mayoritario de los directivos hoteleros, que consideran el cumplimiento de la ley y la presión de las autoridades ambientales como los detonantes de la gestión en las entidades que dirigen.

Los hoteles en la categoría de "líder" sobresalen por un comportamiento homogéneo. Todos aplican más de $83 \%$ de los indicadores evaluados, con excepción del estímulo al uso de productos ecológicos, la publicación de memorias ambientales y el monitoreo de la generación de residuos sólidos y emisiones de $\mathrm{CO}_{2}$, indicadores que no son aplicados en ningún hotel, no solo de esta categoría, sino en general de la totalidad de la muestra estudiada.

Todos los hoteles del grupo "proactivo" cuentan con planes de emergencias, control de sus impactos ambientales negativos y estándares ambientales en sus operaciones, toman en cuenta a sus clientes para la GA y poseen procedimientos de seguridad. Similar desempeño se logra en el monitoreo de agua y energía 
y en los indicadores relacionados con la disposición a cumplir (conocimiento acerca del SGA-RAN y el aval ambiental, su implantación en la empresa o intención de hacerlo, reconocimiento de los beneficios del sGa para el hotel, así como la vinculación y el apoyo recibido de organismos públicos y del gobierno local en materia ambiental).

Adicionalmente a las limitaciones señaladas para el $100 \%$ de la muestra en relación con los indicadores de monitoreo y comunicación, este grupo se caracteriza por la ausencia de información a clientes y proveedores acerca de los riesgos ambientales y de las acciones para que contribuyan a mitigar los impactos ambientales negativos del hotel.

Otros resultados limitados se observan en cuanto a la política ambiental, la capacitación y la aplicación de tecnologías ambientales, indicadores que solo son considerados por $50 \%$ de los hoteles. Los resultados son similares en la realización de auditorías ambientales, vínculos con otras entidades para desarrollar acciones en favor de la naturaleza y la implantación de los SGA.

Las entidades cuyo comportamiento ambiental se clasificó en la categoría de "reactivo" son aquellas cuyos esfuerzos de gestión se concentran en las exigencias de las casas matrices de sus cadenas y la remediación de los problemas señalados por inspecciones de las autoridades ambientales. Sus SGA son en general formales o no se han diseñado, y mayoritariamente no han contado con el financiamiento para acometer muchas de las acciones planificadas para mejorar sus resultados ambientales.

Los directivos opinan que aunque resulta necesaria la protección del ambiente, los procesos de gestión en el hotel no son supervisados con la sistematicidad requerida. Consideran que la solución de los problemas ambientales recae en las autoridades y que sus prioridades son la calidad y la eficiencia financiera. Sus acciones de GA se concentran en la reducción de los consumos de agua y energía y en prácticas de reciclaje no siempre efectivas.

No obstante, todos controlan sus impactos ambientales, poseen planes de emergencia ambiental, operan bajo estándares ambientales y tienen procedimientos de seguridad. Asimismo, monitorean el agua y la energía, conocen el SGA-RAN y el aval ambiental y tienen intención de implantarlos, reciben apoyo de organismos públicos para su GA y establecen vínculos con el gobierno local. 
Estos establecimientos presentan limitaciones adicionales a las encontradas en toda la muestra en los indicadores relacionados con la consideración de sus clientes para la GA y la realización de auditorías ambientales (cumplidos solo por $60 \%$ de ellos), la política ambiental, los vínculos con organizaciones en favor de la naturaleza, la información sobre posibles riesgos y el reconocimiento de los beneficios de los SGA (aplicados por 40 \% del grupo); además, las tecnologías ambientales y la implantación de un sGa son cumplidos solo por $20 \%$ de los hoteles, y la capacitación ambiental por ninguno dentro del grupo.

Los resultados más pobres en general se observan en el grupo "pasivo-indiferente”, que posee una GA incipiente, no tiene definidas las responsabilidades en materia de gestión y sus directivos no se implican en el proceso o lo hacen de forma limitada.

A pesar de ello, todos cuentan con planes de emergencia, monitoreo del agua y la energía, conocen los modelos de gestión del RAN y el aval ambiental, muestran intenciones de implantarlos y establecen vínculos con la autoridades ambientales. Hay que resaltar que en este último indicador los vínculos se circunscriben a las inspecciones realizadas por las autoridades.

Sin embargo, solo $50 \%$ controla sus impactos ambientales, identifica estándares de operación, toma en cuenta las necesidades y comentarios de sus clientes en su GA y reconoce los beneficios del SGA. Otros indicadores ausentes en estos hoteles, además de los señalados para toda la muestra, son la capacitación ambiental, la aplicación de tecnologías ambientales, los vínculos con organizaciones en favor de la naturaleza, la realización de auditorías ambientales, la información a los huéspedes y la comunicación sobre posibles riesgos, así como la implantación de un sGa y la ausencia de relaciones de apoyo por parte de organismos públicos.

\section{Conclusiones}

El estudio fue preliminar, pues la muestra no resulta estadísticamente representativa de los hoteles de tres, cuatro y cinco estrellas del destino Varadero.

Los cuestionarios aplicados y las entrevistas a los directivos permiten afirmar que los establecimientos estudiados presentan como principales fortalezas 
la disposición a cumplir con las regulaciones ambientales, en particular alcanzar o mantener el aval ambiental de las autoridades, así como en las acciones de gestión ambiental implementadas en relación con los planes de emergencia, la aplicación de estándares ambientales y el control de impactos ambientales, mientras que las mayores debilidades están asociadas a la comunicación de los resultados ambientales y a la promoción del consumo responsable. Respecto al monitoreo de los aspectos ambientales, este se limita a los consumos de agua y energía.

Los hoteles calificados como "líder" o "proactivo", según su GA, representan $50 \%$. En el grupo "reactivo" se ubica $36 \%$ de la muestra (el mayor grupo), lo que evidencia que la gestión ambiental aún no es un proceso totalmente consciente. Solo dos hoteles (14 \%) fueron clasificados como "pasivo-indiferente", avalados por su incipiente gestión y la falta de liderazgo, y ninguno como "negativo".

Es importante desarrollar sistemáticamente estudios similares en el destino Varadero, comparar sus resultados con otros destinos del Caribe y ampliar la muestra de estudio hasta valores estadísticamente representativos de su planta hotelera.

\section{Fuentes consultadas}

Ayuso, Silvia (2003). Gestión sostenible en la industria turística. Retórica y práctica en el sector hotelero español. Tesis doctoral. Barcelona: Universidad Autónoma de Barcelona.

Azqueta, Diego, et al. (2007). Introducción a la economía ambiental. Madrid: McGraw-Hill.

De Burgos, Jerónimo y José Céspedes (2004). “Un análisis de las dimensiones de la gestión ambiental en los servicios hoteleros”. Dirección y Organización, 30, 5-15.

Gaceta Oficial de la República de Cuba (1997). Ley 81 del Medio Ambiente. Gaceta Oficial de la República de Cuba, edición extraordinaria, $7,47$.

González, Javier y Óscar González (2005). "Environmental Proactivity and Business Performance: An Empirical Analysis”. OMEGA, 33 (1), 1-15. 
(2008). "Determinantes de la proactividad medioambiental en la función logística: un análisis empírico”. Cuadernos de Estudios Empresariales, $18,51-71$.

Guevara, Alejandro (2005). "Política ambiental en México: Génesis, desarrollo y perspectivas”. Ambiente y Sociedad, 821, 163-175.

Guzmán, Aldo (2005). "La gestión ambiental en el sistema hotelero" [en línea]. Disponible en: http://www.abcformacion.com/contenidos/medio_ambiente.htm [2006, 14 de julio].

Lascurain, Carlos (2006). Análisis de la política ambiental. México: Plaza y Valdés.

Llull, Antoni (2003). Contabilidad medioambiental y desarrollo sostenible en el sector turístico. Tesis doctoral. Islas Baleares: Govern de Les Illes Balears.

López, Laureano (2011). “Aplicación de un índice de riesgo para la Evaluación del Desempeño Ambiental de Empresas Hoteleras”. Avanzada Científica, 14 (1), 11-22.

López, Laureano y Bárbara Ramírez (2011). "Sinopsis de la gestión ambiental aplicada en las empresas hoteleras cubanas”. Avanzada Científica, 14 (1), 47-57.

Marques de Almeida, José Joaquim y María da Conceiçao da Costa (2002). "Gestión medioambiental y auditoría”. Revista de Contaduría y Administración, 205, 37-50.

Mateo, José (2002). Planificación ambiental. La Habana: Universidad de La Habana.

Mercado, Alfonso (2008). “ ¿Conducta limpia? Un estudio del comportamiento ambiental manufacturero en México”, en Rhys Jenkins y Alfonso Mercado (coords.). Ambiente e industria en México. Tendencias, regulación y comportamiento empresarial. México: El Colegio de México, 95-114.

Meyer, Carlos, Daniel Aguilera y Leonardo Boto (2007). Guía de recomendaciones ambientales. Secretaría de Turismo de La Nación. Buenos Aires: Secretaría de Turismo.

Mintur (2012). "Hoteles Varadero, Cuba” [en línea]. Cuba: Ministerio de Turismo. Disponible en: http://es.cubafiesta.net/hotel/lista/hoteles-varadero [2012, 10 de septiembre]. 
Negrao, Rachel (2000). Curso de gestión ambiental. Sauípe: Oficina Regional de Ciencia de la Organización de las Naciones Unidas para la Educación, la Ciencia y la Cultura para América Latina y el Caribe.

oea (2003). Manual Gestión de la Calidad Ambiental [en línea]. Organización de Estados Americanos. Disponible en: http://www.science.oas.org/ oea_gtz/libros/Ambiental/ambiental.htm [2009, 8 de septiembre].

Pereira, Elisabeth (2007). "Indicadores ambientais como sistema de informação contábil” [en línea]. Gestiopolis. Disponible en: http://www.gestiopolis.com/recursos3/docs/fin/indamb.htm [2008, 9 de febrero].

Pérez, M. (2010). Modelo y procedimiento para la gestión integrada y proactiva de restricciones físicas en organizaciones hoteleras. Tesis de Doctorado en Ciencias Técnicas. Holguín: Universidad de Holguín "Oscar Lucero Moya”.

Puente, Y. (2010). Propuesta de una lista de chequeo para la evaluación de la gestión ambiental empresarial. Tesis de Máster en Ciencias. Matanzas: Universidad de Matanzas "Camilo Cienfuegos".

Senior, Alexa, et al. (2007). "Responsabilidad ambiental: factor creador de valor agregado en las organizaciones”. Revista de Ciencias Sociales, 13 (3), 28-37.

Sil, Marina (1998). “La gestión ambiental en México: 1988-1996”, en Américo Saldívar (coord.). De la economía ambiental al desarrollo sustentable (alternativas frente a la crisis de gestión ambiental). México: Universidad Nacional Autónoma de México, 97-120.

Vargas, Elva, Lilia Zizumbo y Carlos Viesca (2011). “Turismo y ambiente. Un estudio de los comportamientos ambientales en el sector hotelero de Cancún”, en David Juárez (ed.). Turismo, economía y medio ambiente. Madrid: Editorial Académica Española, 123-147.

Vidal, César, et al. (1995). "Modelos de morfodinámica de playas”. Ingeniería del Agua, 2 (extraordinario), 55-74.

Yunis, Eugenio (2003). "Sostenibilidad del turismo y el papel de la certificación”. Comunicación de la Conferencia Regional de las Américas, Sauípe, Brasil, 29 y 30 de septiembre. 